\title{
Subjective Sleep Disturbances of Factory Workers in Relation to Shift Work Schedule and Chronotype
}

\author{
Pamela Song ${ }^{1}$, Su Jung Choi ${ }^{2,3}$, Eun Yeon Joo $^{2}$ \\ ${ }^{1}$ Department of Neurology, Inje University College of Medicine, Ilsan Paik Hospital, Goyang, \\ ${ }^{2}$ Department of Neurology, Neuroscience Center, Samsung Medical Center, Sungkyunkwan University School of Medicine, Seoul, and \\ Department of Health Sciences and Technology, SAIHST, Sungkyunkwan University, Seoul, \\ ${ }^{3}$ Department of Nursing, Samsung Medical Center, Department of Clinical Nursing Science, Graduate School of Clinical Nursing Science, \\ Sungkyunkwan University, Seoul, Korea
}

Received August 19, 2016

Revised November 4, 2016

Accepted December 18, 2016

Address for correspondence Eun Yeon Joo, MD, PhD Department of Neurology,

Neuroscience Center,

Samsung Medical Center,

Samsung Biomedical Research

Institute, Sungkyunkwan

University School of Medicine,

Samsung Medical Center,

81 Irwon-ro, Gangnam-gu,

Seoul 06351, Korea

Tel: $+82-2-3410-3597$

Fax: +82-2-3410-0052

E-mail: ejoo@skku.edu
Objectives: Shift work in Korea is most prevalent in automobile system manufacturing industries. The subjective sleep complaints and individual chronotypes of shift worker was evaluated. Methods: A cross-sectional study was conducted among single automobile system manufacturing industries in Korea. All participants completed questionnaires about the work schedule, duration of sustained employment and various division of work. And sleep questionnaires including: Epworth Sleepiness Scale, Insomnia Severity Index, Hospital Anxiety and Depression Scale, and Morningness-Eveningness Questionnaire. Results: This study enrolled 208 participants [shift workers (SWs) $=142$, day workers (DWs)=66]. Insomnia symptoms were significantly worse in the SW group $(p<0.001)$. Excessive daytime sleepiness was not significantly different between groups. The evening chronotype in DWs was related to both excessive daytime sleepiness (EDS) $(p=0.008)$ and insomnia $(p=0.034)$. On the other hand, chronotypes in SWs were not significantly different in terms of EDS and insomnia. Conclusions: Insomnia symptoms were significant in SWs compare to DWs. In DWs the evening types were sleepier and had more insomnia symptoms than other chronotypes. The sleep symptoms are prevalent in automobile industries workers, and in part it is related to work schedule and individual chronotype.

J Sleep Med 2016;13(2):40-45

Key Words: Shift work, Chronotype, Daytime sleepiness, Insomnia.

\section{Introduction}

The globalization of society requires industries to optimize productivity. Shift work allows 24 -h operation for effective use of available natural resources and manpower in terms of costeffective production and round-the-clock services. ${ }^{1}$ Shift work is defined as an arrangement of working hours that uses two or more teams of workers to extend the hours of operation beyond those of conventional office hours. ${ }^{1}$ It is estimated that nearly one-fifth of the total global workforce works in shifts. ${ }^{2}$

Shift workers are vulnerable to circadian misalignment by maintaining alertness during the night and trying to sleep during the day. Circadian rhythm synchronization is essential for optimal health. ${ }^{2,3}$ It has been reported that shift workers are

This is an Open Access article distributed under the terms of the Creative Commons Attribution Non-Commercial License (http://creativecommons.org/licenses/by-nc/3.0) which permits unrestricted non-commercial use, distribution, and reproduction in any medium, provided the original work is properly cited. at higher risk of sleep disturbance and numerous medical conditions, including cardiovascular disease and diabetes mellitus, among others. Excessive daytime sleepiness (EDS) during work and difficulty sleeping when not working is common amongst shift workers, and approximately $10 \%$ of these worker experience disturbances severe enough to warrant medical attention for shift work sleep disorder. ${ }^{4}$

Each individual has variable endogenous circadian rhythms. These inter-individual differences are often divided into two groups: morning types, and evening types. Morning types prefer rising early and achieving maximum mental and physical activity in the morning. In contrast, evening types prefer later-than-average bed and rise times and function at their peak later in the day than do morning types. ${ }^{5}$ However, shift work forces certain work and sleep schedules irrespective of a typical day and night. An unnatural mandatory wake time may be adaptable for some individuals in respect to certain chrono- 
types of morning types or evening types, and this adaptation may play a role in optimizing work in shift workers.

This study examined shift workers in automobile system manufacturing industries, particularly the YS Enterprise Corporation. The company operates 24 hours a day. This automobile industry would be representative sample of the most prevalent shift work force in Korea. In this study, we evaluated sleep disturbance including EDS, insomnia, depressive mood and anxiety, and individual chronotypes according to shift schedules.

\section{Methods}

\section{Participant selection}

A manufacturing company with both shift and day work schedules was selected for this questionnaire-based cross-sectional study. The questionnaires were distributed to the YS Enterprise Corporation, which is located in Chungnam, Korea, in 2015. This company manufactures parts for automobile systems, which employs 728 workers. The work schedule includes day work, two-shift work, three-shift work, and variable shift work. In this study, the shift workers (SWs) group included only two-shift schedule shift workers, which shift work starts either at 8 a.m. or 8 p.m. The day workers (DWs) group included workers with regular working hours from 9 a.m. to 5 p.m.

\section{Clinical assessment}

Each individual was evaluated using a questionnaire that assessed the employees' work schedule, duration of sustained employment and various division of work. Any sleep disturbance was also evaluated with standardized questionnaires. Participants were assessed for EDS using the Epworth Sleepiness Scale (ESS), for insomnia symptoms using the Insomnia Severity Index (ISI), and for depression and anxiety with the Hospital Anxiety and Depression Scale (HADS). Individual chronotypes were further assessed with the Morningness-Eveningness Questionnaire (MEQ).

\section{Epworth Sleepiness Scale}

The ESS measures the impact of subjective daytime sleepiness. ${ }^{6}$ The Korean version of the ESS consists of eight items that assess the participant's likelihood of falling asleep in a particular situation that is commonly encountered in daily life. Each item ranges from 0 (no napping) to 3 (high chance for napping), with total scores ranging from $0-24{ }^{6}$ A total raw score of $>10$ points is considered to indicate EDS. ${ }^{7}$ The reliability of the ESS was Cronbach alpha $=0.80$.

\section{Insomnia Severity Index}

The ISI questionnaire was used to screen symptoms of in- somnia. A Korean version of the 7-item subjectively reported ISI that assessed the severity of insomnia symptoms was used. The ISI queried participant about the sleep problems in the past 2 weeks. Participants graded each question on a scale between minimal ( 0 points) to very severe ( 4 points). The final score ranged from $0-28$ points, with higher numbers indicating the severity of insomnia. An ISI score $\geq 15$ was considered as clinically significant insomnia. ${ }^{8}$ The reliability of the ISI was Cronbach alpha $=0.85$.

\section{Hospital Anxiety and Depression Scale}

The HADS is used to determine the levels of anxiety and depression that a patient is currently experiencing. The HADS is a 14-item scale that generates ordinal data and is divided into seven items related to anxiety (HADS-A) and seven items related to depression (HADS-D). Each item on the questionnaire is scored from $0-3$, which means that a person could score from 0-21 for either anxiety or depression. ${ }^{9}$ The generally accepted cut-off point is 8 or above for both the HADS-A and HADS-D. ${ }^{10}$ Cronbach alpha $=0.88$ for anxiety and 0.82 for depression.

\section{Morningness-Eveningness Questionnaire}

The MEQ is aimed at determining when the respondent's natural propensity to be active lies during the daily temporal span, as well as one's sleep and wake behavior and schedules. The Korean version of the MEQ consists of 19 items pertaining to habitual rising and bed times, preferred times of physical and mental performance and subjective alertness after rising and before going to bed. ${ }^{5,11}$ Five questions use scales divided into 1 -h or 15-min intervals, and 14 questions are measured on a 4-point Likert-type scale. MEQ scores range from 16-86, with higher scores reflecting stronger morning type preference. Five different classifications are derived from the sum of the scores: definitely evening type (16-30), moderate evening type (31$41)$, intermediate type (42-58), moderate morning type (59$69)$, and definitely morning type (70-86). ${ }^{5}$ The reliability of the MEQ was Cronbach alpha $=0.72$.

\section{Statistical analysis}

Statistical analyses were conducted using the Statistical Package for the Social Sciences (SPSS) for Windows, Version 17.0 (SPSS Inc., Chicago, IL, USA). The alpha level for statistical significance was accepted for two-tailed $p$ values $<0.05$. Continuous data are expressed as mean \pm standard deviation and categorical data as frequencies and percentages. All continuous variables were analyzed using the Mann-Whitney U test, and Kruskal-Wallis test due to an uneven distribution, and categorical variables were evaluated using either chi-square tests or Fisher's exact test. 
Table 1. Demographic findings and sleep questionnaires of shift workers and day workers

\begin{tabular}{|c|c|c|c|}
\hline & Shift workers $(n=142)$ & Day workers $(\mathrm{n}=66)$ & $p$ \\
\hline Age, years & $42.44 \pm 7.15$ & $43.09 \pm 5.77$ & 0.168 \\
\hline Sex-male, n (\%) & $142(100)$ & $55(83.3)$ & $<0.001$ \\
\hline Sustained employment, years & $17.57 \pm 5.90$ & $17.37 \pm 6.68$ & 0.552 \\
\hline \multicolumn{4}{|l|}{ Division of work, n (\%) } \\
\hline Manufacture & $86(60.6)$ & $12(18.2)$ & \\
\hline Casting & $47(33.1)$ & $18(27.3)$ & \\
\hline Inspection & $1(0.7)$ & $14(21.2)$ & \\
\hline Maintenance & $1(0.7)$ & $8(12.1)$ & \\
\hline Polishing & $3(2.1)$ & $5(7.6)$ & \\
\hline Others & $4(1.9)$ & $9(4.3)$ & \\
\hline ESS & $13.70 \pm 4.97$ & $12.59 \pm 4.74$ & 0.124 \\
\hline $\mathrm{ESS}>10, \mathrm{n}(\%)$ & $106(75.7)$ & $42(63.6)$ & 0.052 \\
\hline ISI & $16.05 \pm 5.98$ & $12.41 \pm 6.29$ & $<0.001$ \\
\hline ISI $>14, \mathrm{n}(\%)$ & $85(60.3)$ & $26(40.0)$ & 0.005 \\
\hline HADS-A & $9.88 \pm 4.52$ & $10.20 \pm 4.69$ & 0.485 \\
\hline HADS-D & $11.64 \pm 3.65$ & $11.09 \pm 3.96$ & 0.484 \\
\hline
\end{tabular}

ESS: Epworth Sleepiness Scale, ISI: Insomnia Severity Index, HADS: Hospital Anxiety and Depression Scale, A: anxiety, D: depression

Table 2. Shift workers and day workers characteristic by individual chronotype

\begin{tabular}{|c|c|c|c|c|}
\hline & Morning type & Intermediate & Evening type & $p$ \\
\hline \multicolumn{5}{|l|}{ Total } \\
\hline $\mathrm{n}(\%)$ & $24(11.5)$ & $159(76.4)$ & $25(12.0)$ & \\
\hline Age, years & $44.31 \pm 4.33$ & $43.04 \pm 6.59$ & $40.16 \pm 4.54$ & 0.007 \\
\hline Sustained empolyment, years & $18.55 \pm 4.41$ & $17.79 \pm 6.55$ & $14.35 \pm 3.91$ & 0.012 \\
\hline $\mathrm{ESS}$, mean $\pm \mathrm{SD}$ & $11.66 \pm 5.58$ & $13.15 \pm 4.66$ & $16.16 \pm 4.90$ & 0.008 \\
\hline ISI, mean \pm SD & $13.04 \pm 7.15$ & $14.68 \pm 6.14$ & $18.12 \pm 5.45$ & 0.034 \\
\hline \multicolumn{5}{|l|}{ Shift workers } \\
\hline $\mathrm{n}(\%)$ & $15(10.6)$ & $110(77.4)$ & $17(12.0)$ & \\
\hline Age, years & $46.25 \pm 3.59$ & $43.04 \pm 6.04$ & $40.76 \pm 4.27$ & $0.003^{*}$ \\
\hline Sustained work, years & $20.13 \pm 3.97$ & $17.69 \pm 6.17$ & $14.52 \pm 4.15$ & $0.009^{*}$ \\
\hline $\mathrm{ESS}$, mean $\pm \mathrm{SD}$ & $12.33 \pm 6.16$ & $13.51 \pm 4.60$ & $16.11 \pm 5.62$ & 0.170 \\
\hline ESS>10, n (\%) & $9(60)$ & $82(75.9)$ & $15(88.2)$ & 0.177 \\
\hline ISI, mean \pm SD & $15.93 \pm 5.50$ & $15.61 \pm 5.9$ & $19.00 \pm 6.16$ & 0.203 \\
\hline ISI $>14, \mathrm{n}(\%)$ & $9(60)$ & $63(57.8)$ & $13(76.5)$ & 0.343 \\
\hline HADS-A, mean \pm SD & $9.60 \pm 5.22$ & $9.68 \pm 4.3$ & $11.41 \pm 5.26$ & 0.445 \\
\hline HADS-D, mean \pm SD & $10.86 \pm 4.29$ & $11.54 \pm 3.55$ & $12.94 \pm 3.59$ & 0.302 \\
\hline \multicolumn{5}{|l|}{ Day workers } \\
\hline $\mathrm{n}(\%)$ & $9(13.6)$ & $49(74.2)$ & $8(12.1)$ & \\
\hline Age, years & $41.83 \pm 6.08$ & $43.15 \pm 7.54$ & $38.90 \pm 5.12$ & 0.392 \\
\hline Sustained empolymnet, years & $16.02 \pm 4.87$ & $18.22 \pm 7.22$ & $13.97 \pm 3.57$ & 0.381 \\
\hline $\mathrm{ESS}$, mean $\pm \mathrm{SD}$ & $10.55 \pm 4.58$ & $12.36 \pm 4.73$ & $16.25 \pm 3.19$ & $0.034^{*}$ \\
\hline ESS $>10, \mathrm{n}(\%)$ & $3(25)$ & $31(63.3)$ & $8(100)$ & $0.017^{*}$ \\
\hline ISI, mean \pm SD & $8.22 \pm 7.22$ & $12.58 \pm 6.14$ & $16.35 \pm 3.05$ & $0.030^{*}$ \\
\hline ISI $>14, \mathrm{n}(\%)$ & $2(22.2)$ & $18(37.5)$ & $6(75.0)$ & 0.067 \\
\hline HADS-A, mean \pm SD & $8.37 \pm 5.70$ & $10.30 \pm 4.52$ & $11.37 \pm 4.80$ & 0.535 \\
\hline HADS-D, mean \pm SD & $10.25 \pm 5.23$ & $10.89 \pm 3.71$ & $13.12 \pm 4.01$ & 0.298 \\
\hline
\end{tabular}

${ }^{*} p<0.05$. ESS: Epworth Sleepiness Scale, ISI: Insomnia Severity Index, HADS: Hospital Anxiety and Depression Scale, A: anxiety, D: depression 


\section{Results}

\section{Participant characteristics of SWs and DWs}

In all, 217 participants completed the questionnaire, and 9 participants were excluded (one with missing data on work schedule status, seven with an irregular shift work and daytime work schedule, and one with 3-shift system). The study finally enrolled 208 participants [SWs:DWs=142 (68.3\%):66 (31.7\%)] (Table 1). The mean age of participants was not significantly different between groups ( $p=0.168$ ), and both groups had a male predominance. The workers have been employed for more than 17 years in both groups (SWs:DWs $=17.57 \pm 5.90$ : $17.37 \pm 6.68$ years). Total of 87 (40.0\%) participants had only been on a shift work schedule since beginning of employment, and $27(12.4 \%)$ were only on a day work schedule. There were 12 divisions of work, which operates in shift work and fixed daytime work. The majority of participants were on either a

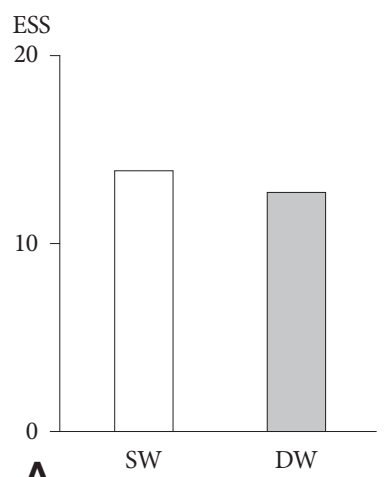

A

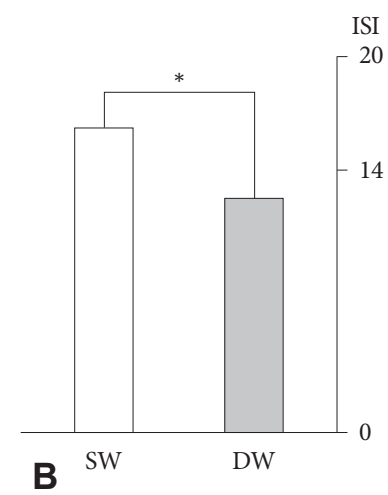

Figure 1. Excessive daytime sleepiness and insomnia symptom severity. (A) ESS is clinically significant in both SWs and DWs (ESS in SWs and DWs). (B) Insomnia symptoms were more common in SWs (ISI in SWs and DWs). ${ }^{*} p<0.05$. ESS: Epworth Sleepiness Scale, ISI: Insomnia Severity Index, SW: shift workers, DW: day workers.

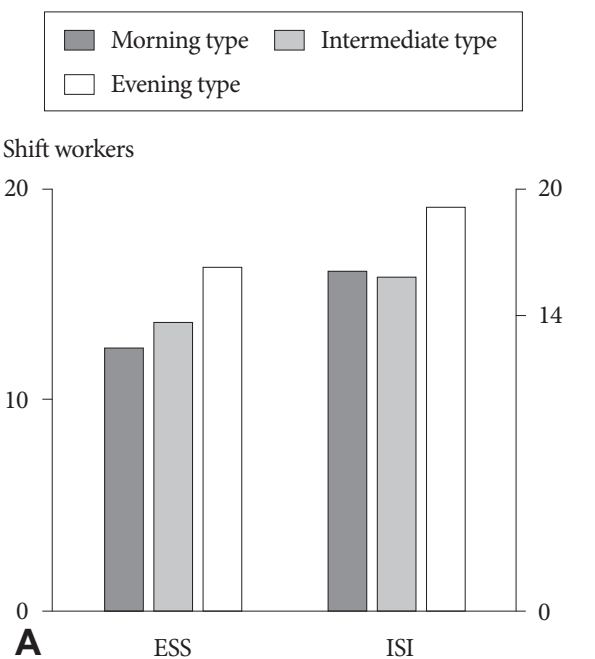

manufacture team $(n=98,47.1 \%)$ or a casting team $(n=65$, $31.5 \%$ ). The division of work revealed that there were more manufacturing teams amongst SWs and more maintenance and inspection teams amongst DWs [manufacture team SW: $\mathrm{DW}=86$ (60.6\%):12 (18.2\%), $p<0.001$; maintenance team SWs: DWs $=1(0.7 \%): 8$ (12.1\%), $p=0.001$; inspection team SWs: DWs $=1(0.7 \%): 14(21.2 \%), p<0.001]$.

\section{Sleep disturbance, mood and chronotype}

Insomnia as a sleep symptom was significantly worse in the SW group (ISI: SWs $16.05 \pm 5.98$, DWs $12.41 \pm 6.29$, $p<$ 0.001 ). Clinically significant insomnia symptoms defined as ISI $\geq 15$, were seen in $60.3 \%$ of SWs and in $40 \%$ of DWs ( $p=$ 0.005 ) (Table 2). Daytime sleepiness were seen in both groups (ESS of SWs vs. DWs $=13.70 \pm 5.98$ vs. $12.59 \pm 4.74, p=0.124$ ) and more than half reported clinically significant EDS defined as ESS $>10$ in both groups [SWs:DWs=106 (75.7\%):42 (63.6\%), $p=0.052$ ] (Fig. 1). Depressive mood evaluated by the HADS-D was not different between groups (SWs $11.64 \pm 3.65$, DWs $11.09 \pm 3.96, p=0.484)$. Anxiety assessed using the HADS-A were not different between groups (SWs $9.88 \pm 4.52$, DWs $10.20 \pm 4.69, p=0.485$ ).

Individual chronotypes were evaluated using the MEQ (Table 2). Among the five ranges of morningness-eveningness, there were morning type $(\mathrm{n}=24,11.5 \%)$, intermediate type $(\mathrm{n}=159,76.4 \%)$ and evening type $(\mathrm{n}=25,12.0 \%)$. Morning type is more prevalent in participants with older age $(p=0.007)$ and longer sustained employment $(p=0.012)$. Evening type is related to higher ESS and ISI score (ESS: $p=0.008$ ) (ISI: $p=0.034$ ).

The sleep disturbances according to chronotypes in SWs and DWs were evaluated (Table 2). Among 143 SWs the distribution of choronotype were morning type $(n=15,10.6 \%)$, intermediate type $(n=110,77.5 \%)$, and evening types $(n=17$,
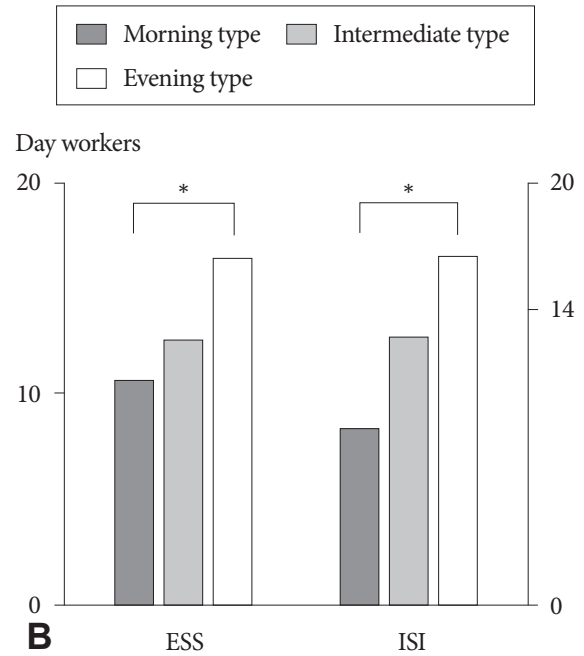

Figure 2. Chronotype and sleep symptoms in SWs and DWs. ESS and ISI according to chronotype (A: SWs, B: DWs). ${ }^{*} p<0.05$. ESS: Epworth Sleepiness Scale, ISI: Insomnia Severity Index, SW: shift workers, DW: day workers. 
12.0\%). The distribution of chronotypes among 66 DWs were morning type $(n=9,13.6 \%)$, intermediate type $(n=49,74.2 \%)$, and evening types $(n=8,12.1 \%)$. The daytime sleepiness and insomnia symptoms were high in all chronotypes of the SWs (ESS: $p=0.170$, ISI: $p=0.203$ ) (Fig. 2). In DWs, evening type had more severe daytime sleepiness and insomnia symptoms (ESS: $p=0.034$, ISI: $p=0.030$ ). Depressive mood and anxiety did not show any significant differences among chronotypes in either the SW or the DW group.

\section{Discussion}

We found significant sleep disturbances in terms of insomnia and possibly for EDS in two-shift schedule in single automobile enterprise in Korea. Previously, sleep disturbances were reported more than $50 \%$ of SWs, compared to only $5-20 \%$ of DWs. ${ }^{12-14}$ The sleep consequences of SW were insomnia symptoms, sleepiness, a shorter sleep time following night work, frequent falling asleep during night work, and having longer nap times. ${ }^{14,15}$ According to Korean Ministry of Employment Survey conducted in 2011, 22\% of manufacturers utilize the shift work policy, with the automobile industry showing the highest ratio at about $44 \% .{ }^{16}$ Considering health consequences, two major domestic automobile industry companies initiated a new two-shift arrangement schedule from 6:30 a.m.-3:10 p.m. and from 3:10 p.m.-12:50 a.m. the next day. This change has eliminated the night shift schedule. However, subcontract enterprises, including this industry, still have trouble improving their shift work schedules. This may have resulted in almost half of participants reporting symptoms of insomnia and EDS, and significant insomnia in SWs.

Shift workers have been reported to be sleepier compared to day workers. ${ }^{17}$ SWs in this study were more frequently distributed in manufacture teams, which production is the main task with high physical labor. According to Korean Ministry of Employment Survey, two-shift schedule leads to 10 extra hours of work each week. ${ }^{16}$ Largely this implies that short total sleep time following shifts, longer working hours, and physical labor may contribute to sleepiness in SWs. However, higher ESS score in SWs did not reveal statistical significance. DWs were as sleepy as SWs. One of the possible explanation may be specific occupation related factors. The workers in automobile enterprise in Korea, regardless of work schedules have poor sleep quality and sleepiness. ${ }^{18,19}$ The evaluation of such factors by our study questionnaire is very limited. Detailed assessment of sleep disorders, underlying medical and psychological disorders, and work and social activity related factors are needed.

An individual chronotype is defined as the phase-relationship between one's sleep-wake cycle and the formal clock time. ${ }^{20}$ According to biological functions in respect to environmental time cues, earlier and later sleep-wake schedules are classified as either morning types or evening types, respectively. The phase difference between extreme early and late chronotypes can be as great as $12 \mathrm{~h},{ }^{21}$ which suggests that an individual's chronotype may influence shift work adaptation. Evening types may experience more sleep disturbances during day shifts, while morning types might report these disturbances more often during night shifts. In particular, it has been suggested that the evening type could lead to better adaptability to shift work, especially better adaptation to night shifts. ${ }^{22,23}$ In this study, evening type day workers reported more insomnia symptoms and daytime sleepiness compared to morning type day workers. On the other hand, shift workers exhibited $>60 \%$ clinically significant insomnia symptoms and daytime sleepiness regardless of their chronotypes. These results were different from our expectations that shift workers with evening types may experience fewer sleep-related symptoms. This difference may be explained by the small number of participants in each chronotype according to work schedule for statistical analysis and also by the non-specific chronotype questionnaire used. The MEQ was designed to assess chronotype in people who work during standard hours, so it has limitations in classifying chronotype in shift workers. In contrast, the Munich Chrono Type Questionnaire for Shift-Workers (MCTQShift) was developed to assess chronotypes in shift workers. ${ }^{24}$ After validation in a Korean population, this type of shift work-specific questionnaire should be used for further study.

Regarding mood symptoms, we expected that SW would result in increased anxiety and depression. This was not supported by our data, as there was no remarkable increase in mood scale by HADS in both SWs and DWs and no significant group differences. Previous studies have investigated the significant relationship between depression, anxiety and SW in particular. ${ }^{17}$ Furthermore, mental health has been reported to be poor in SWs in automobile industries in Korea. ${ }^{19}$

There were several limitations to this study. Our study was based on questionnaires. To assess independent effects of shift work, underlying sleep disorders, underlying medical and psychological disorders, use of sleep related medications or substance, other social and economic factors should be controlled. Especially, the presence of other underlying sleep disorders such as; obstructive sleep apnea syndrome, restless legs syndrome, and other sleep disorders may present symptoms of insomnia and daytime sleepiness. Without these assessment, we cannot clearly state that the results of our study represents independent shift work assessment. Further assessments for shift rotation schedule, rotation speed and direction have not been included. Further on use of shift work non-specific chro- 
notype questionnaire, which MCTQShift have not been validated in Korea, may have affected the results. And small sample size has limited evaluation regarding specific work task related factors. However, this study largely overviews the sleep symptoms and chronotype in two-shift workers compared to day workers in the automobile industry, the most prevalent shift work force in Korea.

\section{Acknowledgments}

This research was supported by Basic Science Research Program through the National Research Foundation of Korea funded by the Ministry of Science, ICT \& Future Planning, Republic of Korea (No. 2014 R1A1A3049510) and by Samsung Biomedical Research Institute grant (\#OTX0002111).

\section{REFERENCES}

1. Pati AK, Chandrawanshi A, Reinberg A. Shift work: consequences and management. Current Science 2011;81:32-52.

2. Sack RL, Auckley D, Auger RR, et al. Circadian rhythm sleep disorders: part I, basic principles, shift work and jet lag disorders. An American Academy of Sleep Medicine review. Sleep 2007;30:1460-1483.

3. Golombek DA, Rosenstein RE. Physiology of circadian entrainment. Physiol Rev 2010;90:1063-1102.

4. Drake CL, Roehrs T, Richardson G, et al. Shift work sleep disorder: prevalence and consequences beyond that of symptomatic day workers. Sleep 2004;27:1453-1462.

5. Horne JA, Ostberg O. A self-assessment questionnaire to determine morningness-eveningness in human circadian rhythms. Int J Chronobiol 1976;4:97-110

6. Cho YW, Lee JH, Son HK, et al. The reliability and validity of the Korean version of the Epworth sleepiness scale. Sleep Breath 2011;15:377-384.

7. Johns MW. A new method for measuring daytime sleepiness: the Epworth Sleepiness Scale. Sleep 1991;14:540-545.

8. Bastien CH, Vallières A, Morin CM. Validation of the Insomnia Severity Index as an outcome measure for insomnia research. Sleep Med 2001; 2:297-307.

9. Zigmond AS, Snaith RP. The hospital anxiety and depression scale.
Acta Psychiatr Scand 1983;67:361-370.

10. Bjelland I, Dahl AA, Haug TT, et al. The validity of the Hospital Anxiety and Depression Scale. An updated literature review. J Psychosom Res 2002;52:69-77.

11. Lee JH, Kim SJ, Lee SY, et al. Reliability and validity of the Korean version of Morningness-Eveningness Questionnaire in adults aged 20-39 years. Chronobiol Int 2014;31:479-486.

12. Akerstedt T. Shift work and disturbed sleep/wakefulness. Occup Med (Lond) 2003;53:89-94.

13. Akerstedt T. Work schedules and sleep. Experientia 1984;40:417-422.

14. Akerstedt T. Shift work and disturbed sleep/wakefulness. Sleep Med Rev 1998;2:117-128.

15. Åkerstedt T. Work hours, sleepiness and the underlying mechanisms. J Sleep Res 1995;4:15-22.

16. Ministry of Employment and Labor. Employment and labor policy in Korea 2011. [cited 2016 Apr 27]. Avilable from URL:http://www.moel. go.kr/view.jsp? cate $=3 \&$ sec $=17 \&$ mode $=$ view $\&$ pimSeq $=1 \&$ piSeq $=1 \& b$ bs_cd $=$ OP0212\&state $=$ A\&seq $=1393132742251$.

17. Bara AC, Arber S. Working shifts and mental health--findings from the British Household Panel Survey (1995-2005). Scand J Work Environ Health 2009;35:361-367.

18. Chun H, Son MA, Kim Y, et al. Effect of shift work on worker's health, family and social life at a automobile manufacturing plant. Korean J Occup Environ Med 1998;10:587-598.

19. Lee JJ, Chung JH. Health assessment of shift workers in a automobile manufacturing plant. Korean J Prev Med 1995;28:103-122.

20. Roenneberg T, Wirz-Justice A, Merrow M. Life between clocks: daily temporal patterns of human chronotypes. J Biol Rhythms 2003;18:80-90.

21. Sehgal A. Methods in Enzymology. Circadian rhythms and biological clocks, part B. Preface. Methods Enzymol 2015;552:xvii-xviii.

22. Hilliker NA, Muehlbach MJ, Schweitzer PK, Walsh JK. Sleepiness/ alertness on a simulated night shift schedule and morningness-eveningness tendency. Sleep 1992;15:430-433.

23. Juda M, Vetter C, Roenneberg T. Chronotype modulates sleep duration, sleep quality, and social jet lag in shift-workers. J Biol Rhythms 2013; 28:141-151.

24. Juda M, Vetter C, Roenneberg T. The Munich ChronoType Questionnaire for Shift-Workers (MCTQShift). J Biol Rhythms 2013;28:130-140. 